\title{
Distribution of lysophospholipids and metabolism of platelet-activating factor in human follicular and peritoneal fluids
}

\author{
N. Lepage ${ }^{1,3}$, P. Miron ${ }^{2,4}$, R. Hemmings ${ }^{4}$, K. D. Roberts ${ }^{1,3}$ and \\ J. Langlais ${ }^{2,3,4 *}$ \\ Departments of ${ }^{1}$ Biochemistry and ${ }^{2}$ Obstetrics-Gynecology, University of Montreal, \\ ${ }^{3}$ Maisonneuve-Rosemont Hospital Research Center, Montreal, and ${ }^{4}$ Institut de Médecine \\ de la Reproduction de Montréal, Canada
}

\begin{abstract}
The distribution of lysophosphatidylcholine, lyso-platelet-activating factor and plateletactivating factor (PAF) was studied in human plasma and in follicular and peritoneal fluid. In plasma, peritoneal and follicular fluids, $51 \%, 87 \%$ and $89 \%$, respectively, of the total lipids were found in the protein fraction (the density $>1.21$ fraction). Two forms of lysophospholipids were identified in this fraction: one of high affinity and one of low affinity for albumin. The metabolism of PAF in human follicular fluid, peritoneal fluid and plasma was also investigated. PAF-acetylhydrolase activity was found in both peritoneal and follicular fluids which induced a time-dependent hydrolysis of $\left.{ }^{3} \mathrm{H}\right] \mathrm{PAF}$. The half-life of PAF was estimated to be 7-12 $\mathrm{min}$ in plasma, $15-25 \mathrm{~min}$ in peritoneal fluid and approximately $2 \mathrm{~h}$ in follicular fluid. PAF-acetylhydrolase activity in embryo culture media supplemented with $10 \%$ serum was markedly inhibited by addition of commercial serum albumin. When $25 \mathrm{~g}$ albumin $\mathrm{l}^{-1}$ was added, $22 \%$ of $\left[{ }^{3} \mathrm{H}\right] \mathrm{PAF}$ was hydrolysed $\mathrm{h}^{-1}$ compared with $72 \%$ in media without albumin. The concentrations of lysophosphatidylcholine measured in plasma, in follicular and peritoneal fluids were 252,286 and $53 \mu \mathrm{mol} \mathrm{l}^{-1}$, respectively. The distribution of these lysophospholipids and the metabolism of PAF in the female genital tract fluids reported in the present study provide evidence for the involvement of these biologically active lipid mediators in a variety of reproductive processes including sperm-egg interactions and embryonic development.
\end{abstract}

\section{Introduction}

Alterations in lipid metabolism are key events regulating several steps of mammalian fertilization, such as sperm capacitation and acrosome reaction as well as sperm-egg fusion (reviewed by Langlais and Roberts, 1985; Parks and Ehrenwald, 1990; Roldan and Harrison, 1990). Compositional and structural changes in proteins, sterols and phospholipids of capacitated sperm membranes are associated with increased membrane fluidity and permeability, along with increased intracellular calcium concentrations, leading to sperm membrane fusions triggering the acrosome reaction. Among those lipid modifications are sperm cholesterol efflux mediated by human female genital tract proteins (Langlais et al., 1981; Langlais et al., 1988) and the breakdown of sperm phospholipids into fusigenic lysolipids, resulting from activation of phospholipases associated with male gametes (Langlais and Roberts, 1985; Antaki et al., 1988; Guérette et al., 1988; Langlais et al., 1992).

Since it is well known that lipid exchanges occur between cells and extracellular fluid compartments, it is anticipated that

*Correspondence should be addressed to: Jean Langlais, Clinique de fertilité, Hôpital Notre-Dame, 1560 rue Sherbrooke Est, Montréal (Québec) H2L 4M1, Canada.

Received 24 June 1992. the lipid composition and metabolism of female genital tract fluids may also play regulatory roles in sperm motility, capacitation and the acrosome reactions, as well as in later reproductive stages such as embryo development and implantation. However, while there have been reports on changes in cholesterol and phospholipids in oviductal fluids of cows during the oestrous cycle (Killian et al, 1989), few data are available regarding lysophospholipid composition and metabolism in human oviductal, follicular and peritoneal fluids that may contribute to the composition of the milieu where sperm-egg interactions occur in vivo. This report presents data on the distribution and metabolism of lysophospholipids in human plasma and in follicular and peritoneal fluids; the lysophospholipids investigated in this study were lysophosphatidylcholine (LPC), lyso-platelet-activating factor (Lyso-PAF) and its precursor, platelet-activating factor (PAF).

\section{Materials and Methods}

\section{Chemicals and reagents}

All solvents and chemicals were of analytical grade. Radioactive material was purchased from Dupont, MA: $\left(\left[{ }^{14} \mathrm{C}\right]-\right.$ 
palmitoyl)-lysopalmitoyl phosphatidylcholine $\left(\left[{ }^{14} \mathrm{C}\right] \mathrm{LPC}\right) \mathrm{NEC}$ 683, I $\left.{ }^{3} \mathrm{H}\right]$ hexadecyl-2-acetyl-sn-glyceryl-3-phosphorylcholine NET-668, alkyl-2[ $\left.{ }^{3} \mathrm{H}\right]$ acetyl-sn-glyceryl-3-phosphorylcholine NET-910, and $\left.1{ }^{3} \mathrm{H}\right]$ alkyl-sn-glyceryl-3-phosphorylcholine (a mixture of $\mathrm{C} 14-\mathrm{C} 16-\mathrm{C} 18$ alkyl ethers) NET-878. NaCl and EDTA were obtained from Sigma, $\mathrm{MO}$, and $\mathrm{KBr}$, chloroform and methanol were purchased from Fisher, NJ.

\section{Collection of follicular fluid, peritoneal fluid and maternal blood plasma}

Samples of human follicular fluid (FF) and peritoneal fluid (PF) were obtained from patients registered in an in vitro fertilization programme. PF from 29 women was obtained at midcycle during exploratory laparoscopy or during tubal ligation procedures. FF was obtained at the time of laparoscopy from 15 women undergoing oocyte recovery for IVF. Only FF and PF with minimal blood contamination were used for the study. When the volume of packed red blood cells, following centrifugation, was more than $2 \%$ of the fluid volume, the samples were not used in the present study. The potential effects of ovarian stimulation were excluded by obtaining control samples of follicular fluid at the time of exploratory laparoscopy and samples of peritoneal fluid were obtained at the time of oocyte retrieval. Fluids collected in either condition did not show significantly different lipoprotein profiles (data not shown). Blood was collected in tubes containing EDTA. Plasma was obtained following a $10 \mathrm{~min}$ centrifugation at $1000 \mathrm{~g}$. PF and FF were centrifuged for $10 \mathrm{~min}$ at $1000 \mathrm{~g}$ to eliminate residual cells. Fluids were kept at $4^{\circ} \mathrm{C}$ for ultracentrifugation or at $-20^{\circ} \mathrm{C}$ until enzymatic assays.

\section{Density gradient ultracentrifugation}

Heat-inactivated $\left(56^{\circ} \mathrm{C}\right.$ for $30 \mathrm{~min}$ ) human plasma, $\mathrm{PF}$ or $\mathrm{FF}$ was incubated in the presence of either radioactive LPC + PAF or LPC + Lyso-PAF for $30 \mathrm{~min}$ at $56^{\circ} \mathrm{C}$. Radioactive products $\left(88 \mathrm{nmol}\left[{ }^{14} \mathrm{C}\right] \mathrm{LPC} \quad\left(57 \mathrm{mCi} \mathrm{mmol}^{-1}\right)\right), \quad 115 \mathrm{pmol} \quad\left[{ }^{3} \mathrm{H}\right] \mathrm{PAF}$ $\left(56.7 \mathrm{Ci} \mathrm{mmol}^{-1}\right)$ and $\left.175 \mathrm{pmol}^{3} \mathrm{H}\right]$ lyso-PAF $\left(52 \mathrm{Ci} \mathrm{mmol}^{-1}\right.$ ) were evaporated to dryness under a stream of nitrogen in $15 \mathrm{ml}$ plastic tubes (Falcon Labware, Becton-Dickinson, Mississauga, Ontario) and resuspended by sonication in $1 \mathrm{ml}$ of a low density solution $(d=1.006)$ of $\mathrm{KBr}$. After sonication of radioactive lipids, $2 \mathrm{ml}$ of fluids (plasma, peritoneal or follicular fluid) was added with $1 \mathrm{ml} \mathrm{KBr}$ solution $(d=1.006)$ and the mixture was incubated for $30 \mathrm{~min}$ at $56^{\circ} \mathrm{C}$. This incubation period allowed the distribution of labelled lipids in the lipoproteins and lipidbinding proteins in the fluids. Incubation at $56^{\circ} \mathrm{C}$ also prevented the hydrolysis of $\left[{ }^{3} \mathrm{H}\right] \mathrm{PAF}$ by PAF-acetylhydrolase present in the fluids (Farr et al., 1983). At the end of the incubation, the density of the mixture was raised to 1.21 by addition of solid $\mathrm{KBr}$ and submitted to density gradient ultracentrifugation according to the methods of Redgrave et al. (1975) and Langlais ef al. (1988). Centrifugation was performed in cellulose nitrate tubes (Beckman Inc., Palo Alto, CA) in a SW41Ti rotor. A fourdensity discontinuous $\mathrm{KBr}$ gradient was prepared and fractions were collected in plastic Eppendorf tubes by retrieving the gradients from the bottom of the cellulose tubes. Radioactivity was measured in each fraction to determine the relative distribution of each of the three lipids in lipoproteins (high density lipoproteins (HDL), low density lipoproteins (LDL) and very low density lipoproteins (VLDL) and proteins (albumin). The lipoprotein elution profile was determined by measuring total cholesterol concentrations in the eluted fractions.

\section{Cholesterol profile}

This profile was obtained using a commercial kit, cholesterol C-system (237574, Boehringer Mannheim, $\mathrm{GmbH}$ ) as described by Langlais et al. (1988). This technique allows the estimation of the total cholesterol concentration (free and esterified forms).

\section{Protein profiles}

The protein fraction $(d>1.21)$ containing albumin was further characterized using a molecular-sieve column (protein column I-125: Waters, Millipore, Milford, MA) coupled to an HPLC system to identify those proteins associated with the radioactive lipids. Before application of samples to the HPLC column, all samples were filtered on $0.2 \mu \mathrm{m}$ filters (Millipore). A phosphate buffer was used to elute the lipid-binding proteins (albumin). The protein fraction was also studied by chromatography on a quaternary methylamine-column (QMA: J. T. Baker, NJ). Elution of proteins was achieved using a discontinuous $\mathrm{NaCl}$ gradient from 0 to $1 \mathrm{~mol} \mathrm{I}^{-1}$, diluted in $20 \mathrm{mmol}$ Tris- $\mathrm{HCl}^{-1}, \mathrm{pH}$ 7.4. Albumin was eluted with $0.2 \mathrm{~mol} \mathrm{NaCl}^{-1}$. A radioactive lipid fraction in its free form or with low affinity for albumin was also absorbed by both types of column and eluted by the addition of methanol, with a continuous gradient for the molecular-sieve column or with the addition of $100 \%$ methanol for the QMA column.

\section{PAF-acetylhydrolase activity}

Determination of PAF-acetylhydrolase (PAF-AH) activity was carried out using $\left[{ }^{3} \mathrm{H}\right]$ acetyl $\mathrm{PAF}$ as the radioactive substrate. This substrate was diluted in $100 \mu \mathrm{l}$ of 0.1 mol Hepes $\mathrm{I}^{-1}$ containing $2 \%$ human albumin (Sigma), $\mathrm{pH}$ 7.2. The enzymatic assay was performed by incubation of $50 \mu \mathrm{l}$ of $\mathrm{PF}$, FF or plasma with $100 \mu \mathrm{l}$ of $0.1 \mathrm{~mol}$ Hepes $1^{-1}$ and $100 \mu \mathrm{l}$ of ${ }^{3} \mathrm{H}[\mathrm{PAF}]\left(10^{5}\right.$ d.p.m.), specific activity, $56.7 \mathrm{Ci} \mathrm{mmol}^{-1}$, in polypropylene tubes. The enzymatic reaction was performed as previously described (Letendre et al., 1992).

To evaluate whether the degradation results were obtained via the activity of PAF-AH, a calcium-independent enzyme (Sturk et al., 1989), and not by calcium-dependent phospholipase $A_{2^{\prime}}$ experiments in the presence and absence of $0.25 \mathrm{~mol}$ EDTA $I^{-1}$ were performed. For this purpose, $50 \mu$ l of fluids, $100 \mu \mathrm{l}$ of $\left[{ }^{3} \mathrm{H}\right] \mathrm{PAF}$ and $100 \mu \mathrm{l}$ of $0.1 \mathrm{~mol}$ Hepes $1^{-1}$ plus $2 \%$ albumin and 0.25 mol EDTA $~^{-1}$ were used as the reaction mixture. The procedure used was as described above.

\section{LPC concentrations}

Total lipids were extracted twice from fluids using a modified Folch extraction as described by Langlais and Roberts (1985). LPC was isolated by applying PF, FF or plasma lipid extracts to silica gel (silica gel $250 \mu \mathrm{m}$, Whatman) thin-layer chromatography (TLC) with the solvent system chloroform:methanol:water 
(65:25:4). In human samples, a region migrated with an $R_{\mathrm{f}}$ value of 0.2 , the same migration as standard LPC or L-PAF. LPC/LPAF was eluted from the silica gel with methanol with a $42 \pm 5 \%$ recovery as determined by addition of $\left[{ }^{3} \mathrm{H}\right] \mathrm{LPC}$ as an internal standard. $\left[{ }^{3} \mathrm{H}\right] \mathrm{LPC}$ (specific activity, $30 \mathrm{Ci} \mathrm{mmol}^{-1}$ ) was prepared by enzymatic hydrolysis of $\left[{ }^{3} \mathrm{H}\right]$ phosphatidylcholine $\left(\left[{ }^{3} \mathrm{H}\right]\right.$ choline) NET-654 (Dupont) and by purification of the $\left.{ }^{3} \mathrm{H}\right] \mathrm{LPC}$ by TLC. The LPC/L-PAF concentration in PF, FF or plasma was obtained via the measurement of inorganic phosphorus (Duck-Chong, 1979) in the eluates, using commercial LPC for determination of the standard curve. Corrections were made to account for losses during extraction procedures; radioactivity was assayed in the eluates before inorganic phosphorus measurement to evaluate the recovery of the internal standard $\left(\left[{ }^{3} \mathrm{H}\right] \mathrm{LPC}\right)$ and to adjust the LPC concentrations to values corresponding to $100 \%$ recovery of $\left[{ }^{3} \mathrm{H}\right] \mathrm{LPC}$.

\section{Results}

\section{Lipid distribution in human plasma, follicular and peritoneal fluids}

The lipoprotein profile in human plasma and follicular fluid was similar to that described by Langlais et al. (1988); LDL, VLDL and HDL were the major cholesterol transporting lipoproteins in plasma, whereas follicular fluid contained essentially a single class of lipoprotein, namely HDL (Figs 1a, b). The distribution of radioactive LPC, L-PAF and PAF in plasma was identical for all three lipids investigated. The lipids were associated with the protein fractions (albumin) and lipoproteins, with 51, 19,19 and $8 \%$ of the radioactivity associated with albumin, HDL, LDL and VLDL, respectively (Fig. 1a).

In follicular fluid, the lysophospholipids and PAF exhibited an identical distribution; $89 \%$ of the radioactivity was associated with the albumin fraction and the remainder with the HDL fraction (Fig. 1b).

Qualitatively, PF contained the same lipoproteins (HDL, LDL, VLDL) as are found in plasma (Fig. 1c). However, the proportion of lipoproteins in the two fluids was different. Approximately the same quantity of cholesterol was found in the HDL fraction of each fluid, whereas there was a mean fivefold decrease in LDL and a mean threefold decrease in VLDL in PF compared with plasma. The distribution of labelled LPC, L-PAF and PAF was the same for all three lipids in this fluid: $87 \%$ of the total radioactivity was found in the protein fractions and the remainder distributed between the lipoproteins (Fig. 1c).

As a high percentage of labelled lipids was associated with the protein fractions, the identification of the lipid-binding proteins was undertaken by applying the protein fractions to a molecular sieve column coupled to an HPLC apparatus. A radioactive profile of the lipids in these eluates was carried out to detect these proteins. The profile obtained for LPC shows that LPC is bound only to human albumin (Fig. 2). The total radioactivity was never recovered when this system of elution with phosphate buffer was used. Thus, it was suspected that a fraction of the lipids was not bound tightly to albumin but was present either as a soluble unbound form or as a form weakly bound to albumin. This fraction of lipid was finally eluted with the addition of methanol (Fig. 2). A methanol gradient performed after the elution of albumin was sufficient to elute the

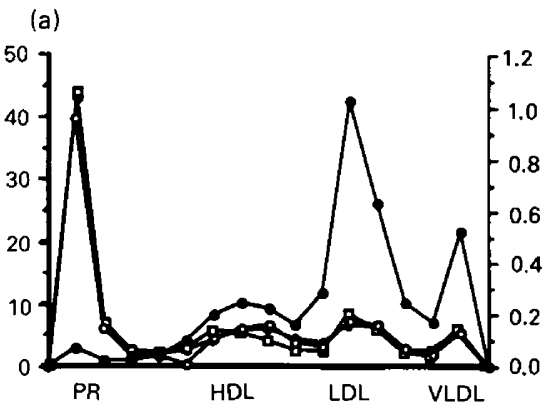

(b)

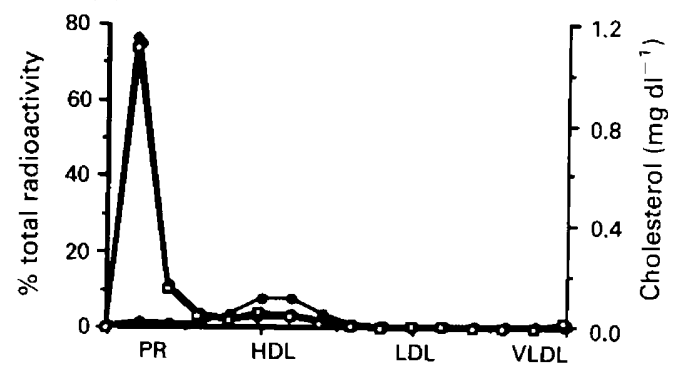

(c)

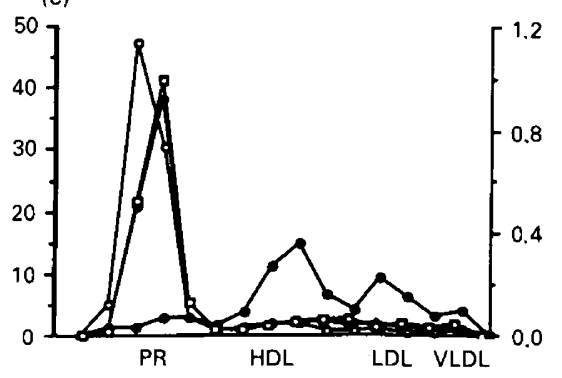

Fig. 1. Distribution of lysophosphatidylcholine (LPC) $(\diamond)$, lysoplatelet-activating factor (L-PAF) $(O)$ and platelet-activating factor (PAF) ( $\square$ ) in (a) human plasma, (b) human follicular and (c) human peritoneal fluids. The protein (PR) and lipoprotein fractions (HDL, LDL, VLDL) are illustrated by the cholesterol profile $(-)$. The distribution of labelled LPC, L-PAF and PAF was determined by measurement of radioactivity. These data represent the means of (a) six different plasma samples (SEM \pm 0.115 ), (b) four samples of FF (SEM \pm 0.04 ), (c) five different samples of fluid (SEM of 0.036).

form of lipid that was not bound to albumin from the column. The same results were obtained for human plasma (data not shown).

A second series of experiments was performed to establish the presence of the two forms of lipid using a quaternary methylamine column to confirm that a soluble unbound form of the lysophospholipid fraction was eluted with methanol (Fig. 3). Albumin saturated with $\left[{ }^{14} \mathrm{C}\right] \mathrm{LPC}$ was eluted with $0.2 \mathrm{~mol}$ $\mathrm{NaCl}^{-1}$ and the radioactivity eluted exactly with the albumin fraction (Fig. 3a). $\left[{ }^{3} \mathrm{H}\right] \mathrm{L}$ yso-PAF and $\left[{ }^{14} \mathrm{C}\right] \mathrm{LPC}$, in the absence of albumin, were then applied to this column and eluted; these unbound forms were eluted only in the presence of methanol (Fig. 3b). Finally, a mixture of albumin saturated with $\left[{ }^{14} \mathrm{C}\right] \mathrm{LPC}$ and of a free form of $\left[{ }^{3} \mathrm{H}\right]$ Lyso-PAF was added to the column and eluted. This allowed the elution of a form of lipid bound to albumin and a form that was eluted with methanol (Fig. 3c). Thus, it would appear that there are two forms of lysophospholipid and PAF in the two fluids tested (FF and human plasma): a 


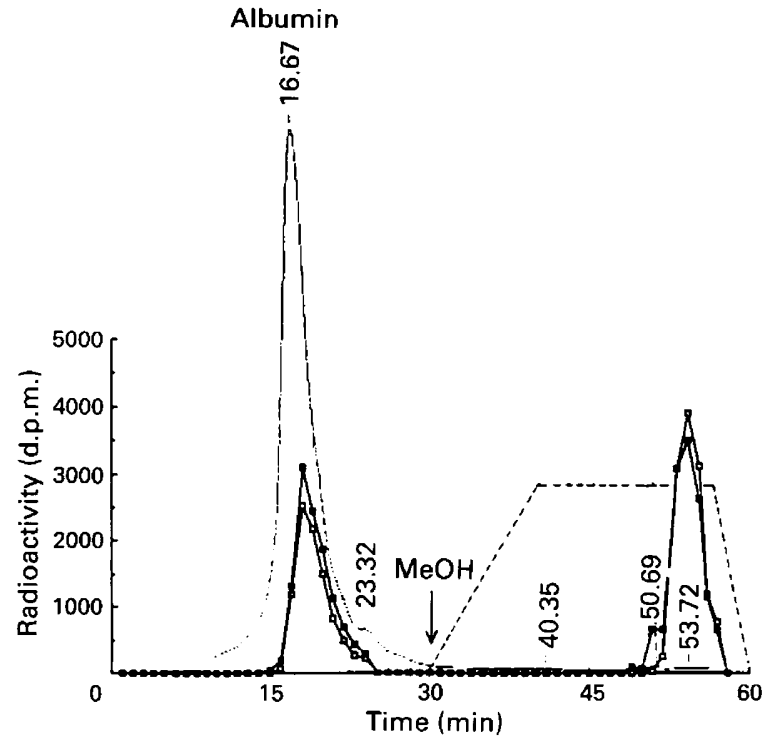

Fig. 2. Molecular weight determination of lysophospholipid-binding proteins in human follicular fluid by HPLC. Proteins were eluted with a phosphate buffer, followed by a linear methanol gradient performed from $0 \%$ to $100 \%$ methanol. ( $\square$ ) $\left[{ }^{3} \mathrm{H}\right]$ platelet-activating factor; $\left[{ }^{14} \mathrm{C}\right]$ lysophosphatidylcholine.

form tightly bound to albumin and a form weakly bound to albumin or possibly free in the biological fluid.

\section{PAF-acetylhydrolase activity in human fluids, follicular and peritoneal fluids}

The enzymatic activity responsible for the hydrolysis of PAF in human plasma, follicular and peritoneal fluids was resistant to freezing at $-20^{\circ} \mathrm{C}$ for several months (data not shown). Thus, PAF-AH activity could be measured either in frozen and thawed samples or in freshly collected fluids without altering the results.

Hydrolysis of $\left[{ }^{3} \mathrm{H}\right] \mathrm{PAF}$ in follicular fluid was markedly lower than in plasma or peritoneal fluid (Fig. 4). The kinetics of PAF hydrolysis in PF were similar to those observed in plasma, except that the activity was significantly lower in peritoneal fluid. In a typical experiment, the percentage of $\left[{ }^{3} \mathrm{H}\right] \mathrm{PAF}$ hydrolysed after incubation for $15 \mathrm{~min}$ in plasma, $\mathrm{PF}$ and $\mathrm{FF}$, was $65 \%$, $39 \%$ and $10 \%$, respectively. The estimated half-life of PAF was 7-12 min in plasma, $15-25 \mathrm{~min}$ in PF and $2 \mathrm{~h}$ in FF.

Since PAF-AH in plasma is associated with lipoproteins (Stafforini et al., 1987, 1989), the specific activity of the enzyme is reflected by the PAF-AH: cholesterol ratio. The mean concentrations of cholesterol in plasma, PF and FF, were 214, 47 and $35 \mathrm{mg} \mathrm{dl}^{-1}$, respectively; thus, the estimated specific activities of PAH-AH during a 15 min incubation in plasma, PF and FF, were $0.30,0.82$ and 0.28 , respectively. The specific activity of $\mathrm{PAH}-\mathrm{AH}$ in follicular fluid was not significantly different from that of plasma, and the lower activity in FF is probably the result of the absence of LDL in this fluid. However, in spite of the low concentration of lipoproteins, peritoneal fluid exhibited a 2.5-fold higher specific activity of PAF-AH compared with that of plasma. This high specific activity of PAH-AH in PF

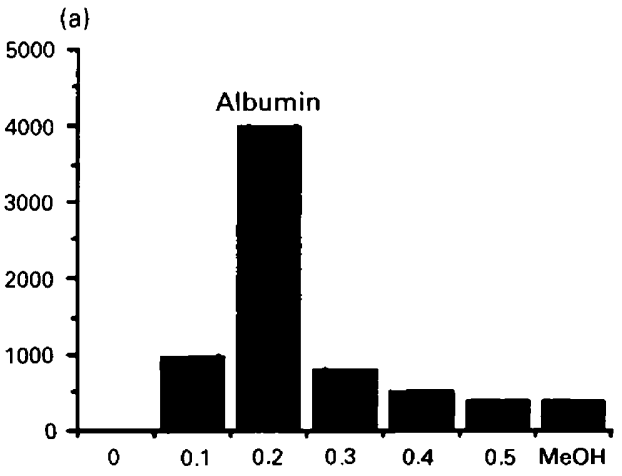

(b)

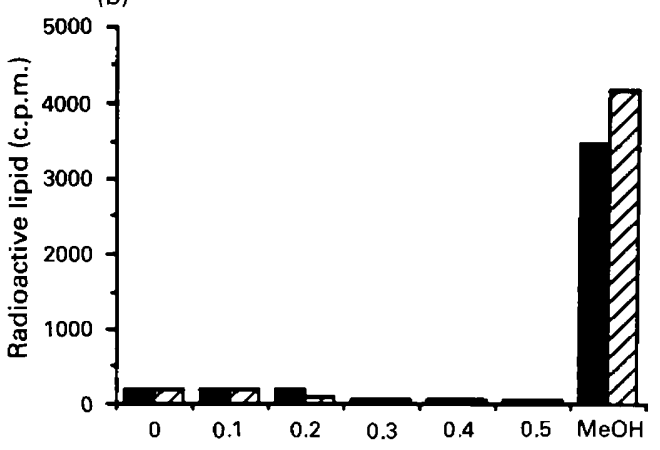

(c)

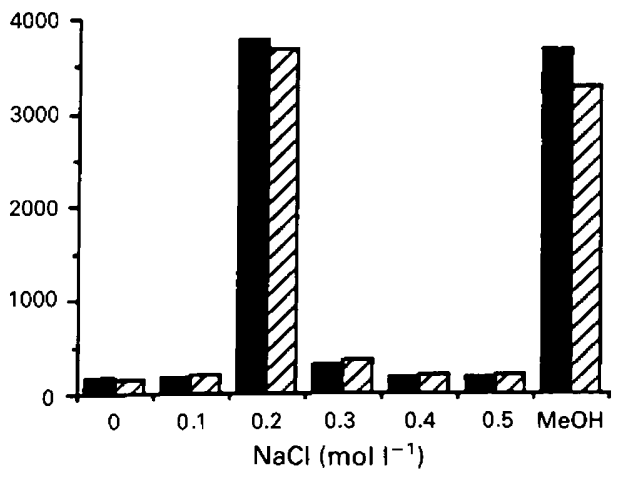

Fig. 3. Elution of lipids associated with albumin and of lipids in a free form (or with low-affinity for albumin) from a quaternary methylamine (QMA) column. ( $\left.{ }^{14} \mathrm{C}\right]$ lysophosphatidylcholine (LPC); (『) [ $\left.{ }^{3} \mathrm{H}\right]$ lysoplatelet-activating factor (Lyso-PAF). (a) Elution of albumin-bound $\left[{ }^{14} \mathrm{C}\right] \mathrm{LPC}$ on QMA. Albumin was eluted with $0.2 \mathrm{~mol} \mathrm{NaCl} \mathrm{1}^{-1}$. (b) Elution of free $\left[{ }^{3} \mathrm{H}\right]$ Lyso-PAF and $\left[{ }^{14} \mathrm{C}\right] \mathrm{LPC}$ on QMA. Lipids were eluted with $100 \%$ methanol. (c) Separation of albumin-bound and weakly-bound or free forms of $\left[{ }^{3} \mathrm{H}\right] \mathrm{L}$ yso-PAF and $\left[{ }^{14} \mathrm{C}\right] \mathrm{LPC}$ on QMA.

might result from altered substrate composition, the presence of enzyme activators or a local increased enzyme content in peritoneal fluid.

Incubation of $\left[{ }^{3} \mathrm{H}\right] \mathrm{PAF}$ in plasma, $\mathrm{PF}$ or $\mathrm{FF}$ in the presence of EDTA did not affect the rates of PAF hydrolysis (data not shown), suggesting that the PAF-AH activity in these fluids is independent of calcium and that PAF hydrolysis would not be mediated by calcium-dependent phospholipase $A_{2}$ in these fluids.

Since PAF was mostly associated with albumin in FF and PF (Fig. 2), the effect of albumin concentration on the rate of 


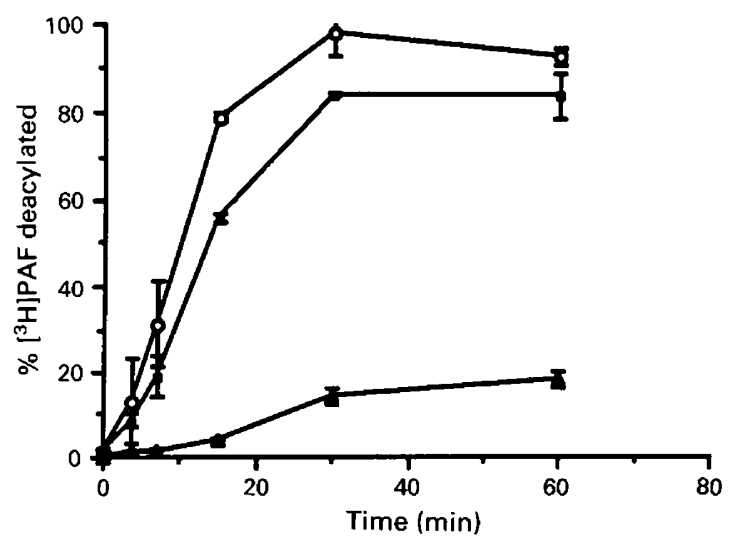

Fig. 4. Kinetic analysis of platelet-activating factor (PAF)acetylhydrolase activating in $(\boldsymbol{\Delta})$ human follicular fluid $(\mathbf{F F}) ;(\boldsymbol{\square})$ human peritoneal fluid (PF); and (O) human plasma. In these fluids, 6, 23 and 30 samples were tested, respectively.

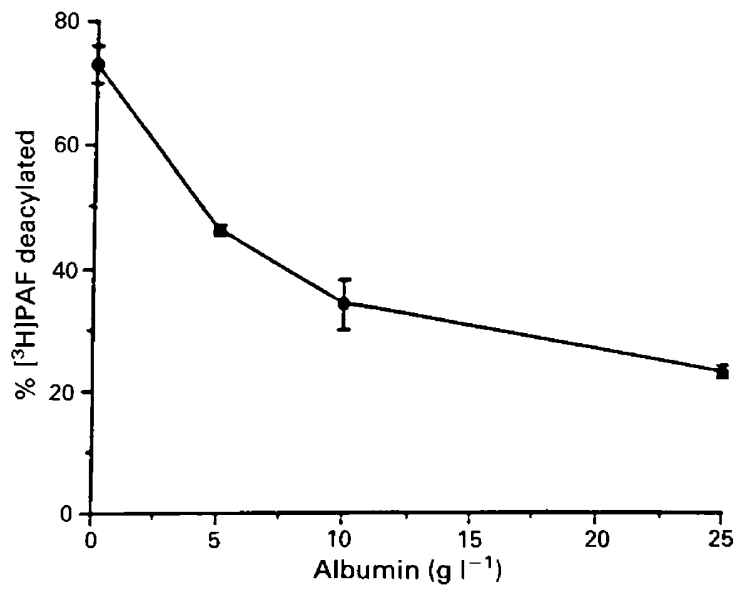

Fig. 5. Effect of albumin on platelet-activating factor (PAF)acetylhydrolase activity in Ham's F-10 supplemented with $10 \%$ human plasma. Exogenous albumin concentrations range from 0 to $25 \mathrm{~g} \mathrm{l}^{-1}$.

PAF hydrolysis in culture media was measured in vitro. A culture medium composed of Hams's F-10 supplemented with $10 \%$ human plasma, hydrolysed $72 \%$ of $\left[^{3} \mathrm{H}\right] \mathrm{PAF}$ during a $\mathbf{1 h}$ incubation (Fig. 5). Addition of exogenous albumin markedly decreased the hydrolysis of PAF, as $25 \mathrm{~g}$ exogenous albumin $\mathrm{I}^{-1}$ resulted in $22 \%$ of PAF hydrolysis $\mathrm{h}^{-1}$.

In view of the reports on the role of PAF in embryonic growth, this increase in the half-life of PAF in culture media supplemented with albumin may be beneficial for the in vitro development of human embryos.

\section{LPC concentrations in human plasma, follicular and peritoneal fluid}

The analysis of inorganic phosphorus associated with the lysophospholipids isolated by thin layer chromatography revealed that the mean concentrations of LPC in human plasma $(n=89)$, follicular $(n=15)$ and peritoneal fluids $(n=29)$, were 252,286 and $53 \mu \mathrm{mol} \mathrm{I}^{-1}$, respectively (Fig. 6).

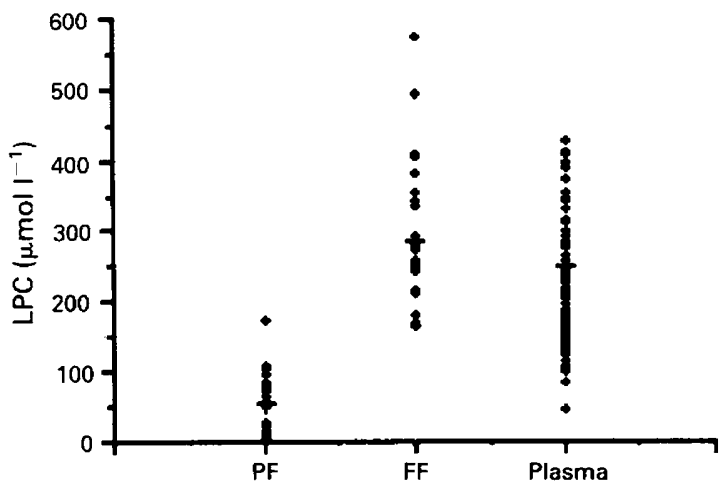

Fig. 6. Lysophosphatidylcholine concentrations in peritoneal fluid (PF), follicular fluid (FF) and human plasma. Concentrations \pm SEM values were $53 \pm 11,286 \pm 18$ and $252 \pm 9 \mu \mathrm{mol}^{-1}$, respectively, in each of the fluids tested.

In the chromatographic system used in this study, LPC and L-PAF comigrate, which means that the LPC concentrations include L-PAF. As plasmalogens represent $5 \%$ of plasma choline phospholipids, it is estimated that the L-PAF contributions to the LPC concentrations reported here do not exceed $1 \%$ of the lysophospholipid concentrations.

\section{Discussion}

Follicular fluid, which surrounds the preovulatory oocyte, accompanies the female gamete at the time of ovulation and may contribute to the composition of the fluids at the locus of fertilization. Thus, human sperm-egg interactions in vivo may occur in a milieu composed of follicular fluid, peritoneal fluid and oviductal secretions. The relative contributions of each fluid may depend upon the time after ovulation and the site at which spermatozoa encounter the oocyte. Presumably, follicular fluid concentrations may form an increasing gradient of chemotactic and capacitation factors that will orient and drive spermatozoa towards the cumulus cells and, ultimately, to the zona pellucida, the site of the physiological acrosome reaction.

Sperm capacitation and the acrosome reaction are membrane phenomena involving exchange of lipids between the sperm plasma membrane and lipid-binding proteins in the capacitation medium. Thus, the results presented in this study concerning the composition, distribution and metabolism of LPC, L-PAF and PAF in human female reproductive tract fluids may provide further insight into the involvement of these biologically active lipids in reproduction and fertility.

The distribution of cholesterol among lipoproteins in human plasma and follicular fluid, observed in the present study, corroborate earlier reports demonstrating that $\mathrm{HDL}$ is the sole class of lipoprotein found in follicular fluid (Simpson et al., 1980; Perret et al., 1985; Langlais et al., 1988). Owing to the molecular-sieve properties of the blood-follicle barrier (Shalgi et al., 1973), high-molecular-weight macromolecules such as LDL and VLDL do not transudate from the blood circulation into follicular fluid. The highest density subclass of HDL particles is conveyed by follicular fluid in which the HDL-cholesterol content is approximately half of that found in plasma (Langlais et al., 1988). A lipoprotein profile somewhat similar to that of 
plasma was observed in peritoneal fluid which displayed comparable concentrations of HDL-cholesterol but lower concentration of LDL and VLDL, suggesting that the blood-peritoneal barrier allows the passage of very high molecular weight species.

The distribution of LPC, L-PAF and PAF among proteins and lipoproteins of female genital tract fluids was studied by incorporating radioactive phospholipids into the fluids for $30 \mathrm{~min}$ at $56^{\circ} \mathrm{C}$, followed by separation of radioactive lipid-binding proteins and lipoproteins by density-gradient ultracentrifugation, molecular sieving on HPLC and ion exchange chromatography. Given the solubility of LPC, L-PAF and PAF in water, the radioactive forms of these lipids would readily achieve equilibrium with endogenous lipids without significantly changing their endogenous concentrations. Thus, it is considered that the elution profiles presented in this study reflect the endogenous distribution of LPC, L-PAF and PAF in human plasma, as well as in follicular and peritoneal fluids.

The labelled lysophospholipids and PAF displayed identical binding patterns in the elution profiles, which is not surprising in view of their very similar acylated molecular structures. In plasma, approximately $51 \%$ of the total radioactivity eluted in the high density $(d>1.21)$ fractions, mostly comprising albumin, while the remainder of the radioactivity was evenly distributed among HDL, LDL and VLDL. These results are in agreement with a previous report on the binding properties of LPC in human plasma (Portman and Illingworth, 1973), indicating that LPC is found not only in the $d>1.21$ fraction (albumin) but also with HDL, LDL and VLDL which exhibit high binding affinities for LPC. The study reported here indicates that L-PAF and PAF follow the same binding distribution as that observed for LPC.

The distribution profile of LPC, L-PAF and PAF in follicular and peritoneal fluids revealed that more than $89 \%$ of the lipids were associated with the albumin fraction, and that the remainder were associated with HDL. This higher proportion of lysophospholipids associated with albumin in follicular fluid is consistent with the reduced binding capacity of low lipoprotein concentrations in this fluid resulting mainly from the absence of LDL. This distribution is also in agreement with a study indicating that LPC accumulates in human follicular fluid mainly in the albumin fraction in which LPC accounts for the major part of the phospholipid composition (Perret et al., 1985). According to the distribution and endogenous concentrations of LPC in human plasma $\left(252 \mu \mathrm{mol} \mathrm{l}^{-1}\right)$ and follicular fluid $\left(286 \mu \mathrm{mol} \mathrm{l}^{-1}\right)$, as reported in the present study, it can be estimated that the average LPC:albumin molar ratios in plasma and follicular fluid are 0.18 and 0.34 .

It was established that albumin was the major LPC-binding protein in follicular fluid by application of the $d>1.21$ fraction on a molecular sieve coupled to HPLC. Only one peak of radioactivity eluted with a retention time identical to that of commercial serum albumin; however, some residual radioactivity was absorbed by the column and labelled lipids could be eluted only by washing the column with methanol. This suggested that LPC was bound to albumin at a high-affinity binding site, but that LPC could also exist either in a soluble free state or in association with albumin via low-affinity binding sites. These results were confirmed by another series of experiments performed with a quaternary methylamine column from which
LPC-albumin and L-PAF-albumin complexes were eluted with $0.2 \mathrm{~mol} \mathrm{NaCl} \mathrm{l}^{-1}$, whereas free LPC and L-PAF were retained and eluted with methanol. Albumin has one high-affinity binding site for LPC (Klopfenstein, 1969) and several binding sites with a low affinity for LPC (Portman and Illingworth, 1973). Thus, it would appear that LPC and L-PAF are bound to follicular fluid albumin via high- and low-affinity binding sites.

The concentrations of LPC in follicular fluid probably reach maximum values at the time of ovulation, since LCAT, which generates LPC via cholesterol esterification on HDL particles, is active in human follicular fluid (Langlais and Roberts, 1985). By acting upon choline plasmalogens, LCAT is probably also responsible for the production of L-PAF in follicular fluid; the endogenous concentration of L-PAF in follicular fluid was not measured in the present study, but it is estimated that it could be similar to that of plasma, which is $125 \mathrm{ng} \mathrm{ml}^{-1}$ or $0.25 \mu_{\mathrm{mol} \mathrm{I}}{ }^{-1}$ (Leonelli et al., 1989). The metabolism of LPC in human follicular fluid in vitro does not result in any significant hydrolysis of LPC (J. Langlais, unpublished observations) since follicular fluid does not contain any red cells which harbour the enzymes (lysophospholipases and acyltransferases) that metabolize LPC in serum.

Thus, the follicle appears to provide an environment that favours LPC accumulation, as evidenced in the present study by the high concentrations of LPC in follicular fluid which may reach concentrations as high as $600 \mu \mathrm{mol} 1^{-1}$, yielding LPC: albumin ratios close to unity.

The observation that albumin of follicular fluid carries twice as much LPC as plasma albumin, suggests that albumin of follicular fluid may have different properties in vivo than those observed with serum albumin in vitro with regard to sperm motility and sperm-egg interactions. Indeed, a report by Jarvi $e t$ al. (in press) indicates that both LPC and L-PAF increase linear and curvilinear velocities of human spermatozoa, and that this effect requires the presence of albumin. This result is consistent with a study showing that follicular fluid enhances sperm motility and velocity in vitro (Falcone et al., 1991). Kyono et al. (1984) showed that the addition of LPC to an in vitro human sperm capacitation medium supplemented with albumin, significantly increases the percentage of egg penetration in the hamster zona-free penetration assay.

The high content of LPC associated with follicular fluid might also contribute to the excellent capacitation potential of this fluid by promoting efficient cholesterol efflux from the sperm surface. In this regard, it is documented that LPC potentiates the release of cellular cholesterol by apolipoprotein A1 (Stein et al., 1979). Stein and coworkers proposed that there are low molecular weight protein-phospholipid complexes (less than $100 \mathrm{kDa}$ ) in serum, which can cross the capillary endothelial barrier, in preference to lipoproteins, and promote cholesterol removal from peripheral cells. This model could represent an additional mechanism underlying the sterol efflux from human spermatozoa mediated by follicular fluid (Langlais et al., 1988) and contribute to the lipid transfer activity of human follicular fluid (Ravnick et al., 1990).

Lysophospholipids present in residual follicular fluid entrapped within the cumulus cell masses surrounding the ovulated oocyte could be active mediators of a sperm capacitation gradient increasing towards the zona pellucida. As direct contact is required between albumin and spermatozoa for 
capacitation in vitro (Dow and Bavister, 1989), it is proposed that complexes of albumin, LPC, L-PAF and PAF of follicular origin may stimulate sperm-egg interactions in vivo. After their encounter with these albumin-lipid complexes, male gametes in quest of an ovum would experience enhanced motility and velocity (Jarvi et al., in press). Since LPC and L-PAF are known to be monocyte chemoattractants (Quinn et al., 1991), it is possible that these bioactive lipid mediators could also exert a chemotactic effect on spermatozoa, attracting them through an increasing gradient, towards the surface of the zona pellucida. Upon binding to the zona, prolonged exposure of spermatozoa to albumin and apoprotein-A-LPC complexes would promote sperm cholesterol efflux, allowing capacitated gametes to undergo the acrosome reaction initiated by signals transduced by the zona pellucida.

The low LPC concentrations detected in peritoneal fluid in the study reported here may have a physiological significance. Indeed, the low concentration of LPC in PF could result in an appropriate medium for the extensive survival of spermatozoa, particularly when the male gametes reach the pouch of Douglas before ovulation. After the arrival of the oocyte and its residual follicular fluid, a gradient of albumin-LPC complexes may be formed with the highest concentrations at the surface of the egg investments and the lowest concentrations of LPC, distant from the oocyte, corresponding to LPC concentrations in peritoneal or oviductal fluids.

After fertilization, the composition and metabolism of lipids in oviductal fluids could play a regulatory role in embryonic development. Given the important function attributed to PAF in preimplantation embryo development (reviewed by Harper, 1989; O'Neill, 1991), the results presented in this study, demonstrating the association of PAF with follicular fluid albumin as well as the prolonged half-life of PAF in fluids void of lipoproteins, suggest that follicular and oviductal fluids would provide a favourable milieu for the accumulation of $\mathrm{PAF}$.

The distribution of $\left[{ }^{3} \mathrm{H}\right] \mathrm{PAF}$ among plasma proteins and lipoproteins, as observed in the present study, is in agreement with earlier reports describing the binding of PAF and L-PAF to lipoproteins (Benveniste et al., 1988) and the binding of PAF to albumin (Clay et al., 1990; Matsumoto and Miwa, 1985). Clay and coworkers (1990) demonstrated that PAF binds to albumin at four binding sites and suggested that the receptor active form of PAF is the albumin-PAF complex, rather than free PAF. Kudolo and Harper (1990) confirmed the ability of albumin to bind PAF with such high affinity that BSA-PAF complexes are resistant to enzymatic degradation.

Given the high proportion of $\left[{ }^{3} \mathrm{H}\right] \mathrm{PAF}$ associated with follicular fluid albumin and the low PAF-AH activity due to the absence of LDL and VLDL in follicular fluid, as evidenced in the present study, it is suggested that follicular fluid favours PAF accumulation in the form of albumin-PAF complexes which are resistant to enzymatic degradation and act as receptor active forms of PAF. The presence of PAF in human follicular fluid has already been reported (Amiel et al,, 1990), with concentrations ranging from 600 to $5000 \mathrm{pg} \mathrm{ml}^{-1}$. Thus, PAF concentrations in human follicular fluid may reach concentrations as high as $10 \times 10^{-10} \mathrm{~mol} \mathrm{1}^{-1}$ corresponding to the biological activity of PAF.

Since oviductal fluid is also a blood transudate characterized by the presence of albumin and HDL, and by the absence of
LDL and VLDL (Ehrenwald et al., 1990), it is probable that oviductal fluid, as well as follicular fluid, would favour an increase in the half-life of embryo-derived PAF and allow autocrine secretion of PAF during embryonic development.

By identifying the physiological conditions that extend the half-life of PAF during embryo development in vivo, some insight may be gained towards the preparation of optimal culture media for human embryos in vitro.

Thus, it was shown here that the addition of exogenous albumin to a culture medium supplemented with $10 \%$ serum markedly decreased the hydrolysis of PAF by serum PAF-AH. Interestingly, embryo culture media with a high albumin content, such as Menezo's B2 medium, sustain good embryo development. Because of its low PAF-AH activity, follicular fluid could be used to supplement culture media used in human IVF and embryo transfer procedures. Indeed, improved pregnancy rates with the GIFT procedure have been reported when follicular fluid is used as a sperm capacitation and gamete medium (Fakih and Vijayakumar, 1990).

Increasing our knowledge of the composition and metabolism of lipids in female genital tract fluids where sperm-egg interactions and embryonic development occur may prove rewarding in creating better conditions for human IVF procedures and also in identifying some metabolic lipid disorders which may be related to the pathogenesis of unexplained infertility.

This work was supported by the Medical Research Council of Canada, Fonds pour la Formation de Chercheurs et l'Aide à la Recherche, and l'Institut de Médecine de la Reproduction de Montréal.

\section{References}

Amiel ML, Testart J and Benveniste J (1990) Presence du PAF-acether (paf) dans le liquide folliculare humain Contraception-Fertilité-Sexualité 18 561-562

Antaki P, Langlais J, Ross P, Guérette P and Roberts KD (1988) Evidence of two forms of phospholipase $A_{2}$ in human semen Gamete Research 19 203-214

Benveniste J, Nunez D, Duvier P, Korth R, Bidault J and Fruchart JC (1988) Preformed PAF-acether and lyso-PAF-acether are bound to blood lipoproteins FEBS Letters $226371-376$

Clay KL, Johnson C and Henson P (1990) Binding of platelet-activating factor to albumin Biochimica et Biophysica Acta 1046 309-314

Dow MPD and Bavister BD (1989) Direct contact is required between serumalbumin and hamster spermatozoa for capacitation in vitro Gamete Research 23 171-180

Duck-Chong CG (1979) A rapid sensitive method for determining phospholipid phosphorus involving digestion with magnesium nitrate Lipids 14 492-497

Ehrenwald E, Foote RH and Park JE (1990) Bovine oviductal fluid components and their potential role in sperm cholesterol efflux Molecular Reproduction and Development 25 195-204

Fakih $\mathbf{H}$ and Vijayakumar $\mathbf{R}$ (1990) Improved pregnancy rates and outcome with gamete intrafallopian transfer when follicular fluid is used as a sperm capacitation and gamete medium Fertility and Sterility 53 515-520

Falcone L, Gianni S, Yannez AP, Marchini M, Eppenberger U and Balerna M (1991) Follicular fluid enhances sperm motility and velocity in vitro Fertility and Sterility 55 619-623

Farr RS, Wardlow ML, Cox CP, Meng KE and Greene DE (1983) Human serum acid-labile factor is an acylhydrolase that inactivates platelet-activating factor Federation Proceedings 42 3120-3122

Guérette P, Langlais J, Antaki P, Chapdelaine A and Roberts KD (1988) Activation of phospholipase $A_{2}$ of human spermatozoa by proteases Gamete Research 19 203-214

Harper MJK (1989) Platelet-activating factor: a paracrine factor in preimplantation stages of reproduction Biology of Reproduction 40 907-913 
Jarvi K, Langlais J, Roberts KD and Gagnon C Effect of platelet activating factor (PAF), lyso-PAF and lysophosphatidylcholine (LPC) on sperm motion: importance of albumin for motility stimulation Fertility and Sterility, in press

Killian GJ, Chapman DA, Kavanaugh JF, Deaver DR and Wiggin HB (1989) Changes in phospholipids, cholesterol and protein content of oviductal fluid of cows during the oestrous cycle Joumal of Reproduction and Fertility 86 $419-426$

Klopfenstein WE (1969) Enthalpy change of binding lysolecithin to serum albumin Biochimica et Biophysica Acta 181 323-325

Kudolo GB and Harper MJK (1990) Estimation of platelet-activating factor receptors in the endometrium of the pregnant rabbit: regulation of ligand availability and catabolism of bovine serum albumin Biology of Reproduction 43 368-377

Kyono K, Hoshi K, Saito A, Tsuiki A, Hoshiai H and Suzuki M (1984) Effects of phospholipase $A_{2}$, lysophosphatidylcholine and fatty acids on the acrosome reaction of human spermatozoa Tahoku Journal Experimental Medicine 144 $257-263$

Langlais J and Roberts KD (1985) Molecular membrane model of sperm capacitation and the acrosome reaction of mammalian spermatozoa Gamete Research 12 183-224

Langlais J, Zollinger M, Plante L, Chapdelaine A, Bleau G and Roberts KD (1981) Localization of cholesteryl sulfate in human spermatozoa in support of a hypothesis for the mechanism of capacitation Proceedings of the National Academy of Sciences USA 78 7266-7270

Langlais J, Kan FWK, Granger L, Raymond L, Bleau G and Roberts KD (1988) Identification of sterol acceptors that stimulate cholesterol efflux from human spermatozoa during in vitro capacitation Gamete Research 20 185-201

Langlais J. Chafouleas, JG, Ingraham R and Vigneault N (1992) The phospholipase $\mathrm{A}_{2}$ of human spermatozoa: purification and partial sequence Biochemical and Biophysical Research Communications 182 208-214

Leonelli FM, Leong LL, Sturm MJ, Strophair JA, Clarke GM and Taylor RR (1989) Plasma levels of the lyso-derivative of platelet-activating factor in acute severe systemic illness Clinical Science 77 561-566

Letendre ED, Miron P, Roberts KD and Langlais J (1992) Platelet-activating factor acetylhydrolase in human seminal plasma Fertility and Sterility $\mathbf{5 7}$ 193-198

Matsumoto M and Miwa M (1985) Platelet-activating factor-binding protein in human serum. In Advances in Prostaglandin. Thromboxane and Leukotriene Research Vol. 15 pp 705-706 Eds O Hayaishi and S Yamamoto. Raven Press, New York

$O^{\prime}$ Neill C (1991) A physiological role for PAF in the stimulation of mammalian embryonic development Trends in Pharmacological Sciences 12 82-84
Parks JE and Ehrenwald E (1990) Cholesterol efflux mammalian sperm and its potential role in capacitation. In Fertilization in Mammals pp 155-167 Eds BD Bavister, I Cummings and ERS Roldan. Serono Symposia, Norwell

Perret BP, Parinaud J, Ribbes H, Moatt JP, Pontonnier G, Chap H and Douste-Blazy L (1985) Lipoprotein and phospholipid distribution in human follicular fluids Fertility and Sterility $\mathbf{4 3}$ 405-409

Portman OW and Illingworth DR (1973) Lysolecithin binding to human and squirrel monkey plasma and tissue components Biochimica et Biophysica Acta $32634-42$

Quinn MT, Kondratenko N and Parthasarathy S (1991) Analysis of the monocyte chemotactic response to lysophosphatidylcholine: role of lysophospholipase C Biochemica et Biophysica Acta 1082 293-302

Ravnik SE, Zarutskie PW and Muller CH (1990) Lipid transfer activity in human follicular-fluid - relation to human sperm capacitation Joumal of Andrology 11 216-226

Redgrave TG, Roberts DCK and West CE (1975) Separation of plasma lipoproteins by density-gradient ultracentrifugation Analytical Biochemistry 65 $42-49$

Roldan ERS and Harrison RAP (1990) Molecular mechanisms leading to exocytosis during the sperm acrosome reaction. In Fertilization in Mammals pp 155-167 Eds BD Bavister, J Cummins and ERS Roldan. Serono Symposia, Norwell

Shalgi R, Kraicer P, Rimon A, Pinto M and Soferman N (1973) Proteins of human follicular fluid: the blood follicle barrier Fertility and Sterility 24 429-434

Simpson ER, Rochelle DB, Carr BR and McDonald PC (1980) Plasma lipoproteins in follicular fluid of human ovaries Journal of Clinical Endocrinology and Metabolism 51 1469-1471

Stafforini DM, McIntyre TM, Carter ME and Prescott SM (1987) Human plasma platelet-activating factor acetylhydrolase: association with lipoprotein particles and role in the degradation of platelet-activating factor Journal of Biological Chemistry 262 4215-4222

Stafforini DM, Carter EM, Zimmerman GA, McIntyre TM and Prescott SM (1989) Lipoproteins alter the catalytic behavior of the platelet-activating factor acetylhydrolase in human plasma Proceedings of the National Academy of Sciences USA $862393-2397$

Stein O, Fainaru M and Stein Y (1979) The role of lysophosphatidylcholine and apolipoprotein $A_{1}$ in the cholesterol removing capacity of lipoproteindependent serum in tissue culture Biochimica et Biophysica Acta 574 495-504

Sturk A, Ten Cate JW, Hosford D, Mencia-Huerta JM and Braquet P (1989) The synthesis, catabolism and pathophysiological role of platelet activating factor Advances in Lipid Research 23 219-276 